\title{
Psychological stress and immune function: establishing both acute and chronic psychological stress models in balb/c mice
}

\author{
E. Vázquez, A. Barranco, M. Manzano, M. L. Jiménez and R. Rueda \\ Science \& Technology, R\&D Dept, Abbott Nutrition, Granada, Spain
}

Stress-induced immunological alterations have been considered to be a major cause of increased risk for immune-related diseases such as cancer, autoimmune disorders, food allergy, alteration of mucosal barrier function and susceptibility to infection. Stressors trigger physiological responses involving the hypothalamic-pituitary-adrenal axis (HPA), which is closely associated with the immune system. It is well known that some foods, in addition to contributing essential nutrients, also contain additional components such as prebiotic, probiotic and other functional ingredients that induce a balanced immune system and improve disease resistance and general healthy status. There is a need for appropriate animal models as a tool for testing the potential benefit of some of these functional foods on the HPA-immune system axis.

The aim of the present work was to establish both acute and chronic mouse models for the study of cellular immunity modulation by psychological stress. Female 6-week-old Balb/c mice were used in both stress models ( $n=12$ for each experimental group). The acute stress model comprised for four consecutive days overnight $16 \mathrm{~h}$-restraint with food and water deprivation (RST model). On the other hand, the chronic stressor was based on the natural panic of mice in relation to water; thus, animals were placed on a very small watersurrounded platform for $1 \mathrm{~h} / \mathrm{d}$ for $10 \mathrm{~d}$ (the water-avoidance stress model; WAS). At the end of both experimental schedules mice were killed and serum, small intestine, spleen and thymus were removed. Physiological variables such as body weight, intestinal length and weight, intestinal mucosa and serum corticosterone levels were recorded. The immunological variables determined were spleen and thymus weights, intestinal secretory IgA content, serum ovoalbumin-specific antibody production, number of peritoneal macrophages, number of spleen cells, number of spleen natural killer cells and the expression of the surface spleen cell markers CD45, CD3, CD4, CD8, CD45R/B220, CD16/CD32 and Ia/Ie. Both stress models were found to impair the normal animal status, affecting most of the selected physiological and immunological variables (table 1). In the RST model the body, thymus and small intestine weights, the levels of corticosterone and ovoalbumin-specific antibody in serum and the number of spleen B-, T-, T-helper, CD3-CD4+ and CD3-CD8 + cells were significantly lower in the stressed animals than in the controls $(P<0.05)$. Similarly, in the WAS model the stressed animals showed a lower numbers of spleen T-helper, T-cytotoxic and total T-cells, although the numbers of CD3-CD4+ and CD3-CD8 + cells were significantly higher. In addition, the level of serum corticosterone and thymus weight were significantly lower in stressed animals than in controls.

In conclusion, both psychological stress models significantly affected the normal status of the mice, the RST model (acute) being much more detrimental to the animals than the WAS model, which seems to be an appropriate tool to mimic the psychological stress damage experienced by populations in Western countries.

Table 1. Data of anthropometric measurements and cell counts

\begin{tabular}{|c|c|c|c|c|}
\hline & \multicolumn{2}{|c|}{ WAS model } & \multicolumn{2}{|c|}{ RST model } \\
\hline & Control & Stress & Control & Stress \\
\hline Body weight $(\mathrm{g})$ & $19,76 \pm 1.07$ & $19,86 \pm 1,28$ & $18,95 \pm 0,85$ & $15,91 \pm 1,24 *$ \\
\hline Thymus weight (g) & $0,059 \pm 0,011$ & $0,039 \pm 0,006^{*}$ & $0,038 \pm 0,009$ & $0,016 \pm 0,006^{*}$ \\
\hline Spleen weight $(\mathrm{g})$ & $0,131 \pm 0,02$ & $0,135 \pm 0,017$ & $0,118 \pm 0,02$ & $0,090 \pm 0,0180$ \\
\hline Spleenocytes $\left(\mathrm{n}^{\circ}\right.$ cells $)$ & $93,11 \times 10^{6} \pm 15,91 \times 10^{6}$ & $98,54 \times 10^{6} \pm 16,35 \times 10^{6}$ & $87,87 \times 10^{6} \pm 25,5 \times 10^{6}$ & $50,53 \times 10^{6} \pm 25,83 \times 10^{6 *}$ \\
\hline NK $\left(n^{\circ}\right.$ cells $)$ & $1,68 \times 10^{6} \pm 0,37 \times 10^{6}$ & $2,703 \times 10^{6} \pm 0,82 \times 10^{6} *$ & $3,05 \times 10^{6} \pm 0,71 \times 10^{6}$ & $1,26 \times 10^{6} \pm 0,22 \times 10^{6 *}$ \\
\hline Small intestine mucose weight (g) & $0,662 \pm 0,092$ & $0,659 \pm 0,071$ & $0,759 \pm 0,078$ & $0,562 \pm 0,091^{*}$ \\
\hline
\end{tabular}

Mean \pm SD. $* P<0.001$. 